\title{
Characteristics of Small Pancreatic Carcinoma: Neural Invasion is Associated with Tumor Recurrence
}

\author{
Hideki Izumi ${ }^{*}$, Hisamichi Yoshii ${ }^{1}$, Masaya Mukai', Eiji Nomura1, Naoki Yazawa ${ }^{2}$, \\ Daisuke Furukawa ${ }^{2}$, Yoshihito Masuoka ${ }^{2}$, Misuzu Yamada², Taro Mashiko², \\ Takuma Tajiri' ${ }^{3}$, Toshio Nakagohri², Hiroyasu Makuuchi ${ }^{1}$ \\ ${ }^{1}$ Department of Gastrointestinal Surgery, Tokai University Hachioji Hospital, Tokyo, Japan \\ ${ }^{2}$ Department of Gastrointestinal Surgery, School of Medicine, Tokai University, Kanagawa, Japan \\ ${ }^{3}$ Department of Pathology, Tokai University Hachioji Hospital, Tokyo, Japan \\ Email: *deehii@is.icc.u-tokai.ac.jp
}

How to cite this paper: Izumi, H., Yoshii, H., Mukai, M., Nomura, E., Yazawa, N., Furukawa, D., Masuoka, Y., Yamada, M., Mashiko, T., Tajiri, T., Nakagohri, T. and Makuuchi, H. (2017) Characteristics of Small Pancreatic Carcinoma: Neural Invasion Is Associated with Tumor Recurrence. Journal of Cancer Therapy, 8, 1125-1134. https://doi.org/10.4236/jct.2017.812096

Received: October 16, 2017

Accepted: December 9, 2017

Published: December 12, 2017

Copyright $\odot 2017$ by authors and Scientific Research Publishing Inc. This work is licensed under the Creative Commons Attribution International License (CC BY 4.0).

http://creativecommons.org/licenses/by/4.0/

\begin{abstract}
Background: Pancreatic carcinoma has a poor prognosis. It is important to focus on early detection and treatment for improvement of the prognosis. Understanding the characteristics of TS1 pancreatic carcinoma $(\leq 2 \mathrm{~cm})$ could improve the treatment outcome of pancreatic carcinoma. Methods: Among 444 patients with conventional pancreatic carcinoma who underwent surgery at our facility, the study targeted 65 (14.6\%) with a histopathological diagnosis of TS1 pancreatic carcinoma. We examined 65 cases of TS1 pancreatic carcinoma in reason for hospital visit, examination findings, histopathological findings, and treatment outcomes. Results: The detection rate of TS1 pancreatic carcinoma by ultrasonography was $83.1 \%$ for visualization of the tumor mass and $96.9 \%$ for main pancreatic duct dilatation. The corresponding rates for endoscopic ultrasound were $92.9 \%$ and $100 \%$. With regard to postoperative outcome, $43.1 \%$ of patients had a recurrence; the site of recurrence was local in $24.6 \%$ and hepatic in $9.2 \%$. On multivariate analysis, intrapancreatic neural invasion was an independent risk factor for recurrence (odds ratio, 6.333; 95\% confidence interval, $1.834-21.872 ; p=0.004)$. Conclusions. To screen for TS1 pancreatic carcinoma, the study first examined for main pancreatic duct dilatation by ultrasonography and then conducted a detailed examination with endoscopic ultrasound. Patients with pancreatic neural invasion require careful attention for local recurrence.
\end{abstract}

\section{Keywords}

Early Detection, Recurrence Factor, Small Pancreatic Carcinoma 


\section{Introduction}

Pancreatic carcinoma has an extremely poor prognosis, and early detection and treatment are the only way to improve the prognosis. Egawa et al. [1] reported a 5 -year survival rate of $80.4 \%$ for pancreatic carcinomas detected with a size $\leq 10$ $\mathrm{mm}$, demonstrating that it is important for improvement of the prognosis to find pancreatic carcinomas at an early stage. However, pancreatic carcinomas $\leq$ $10 \mathrm{~mm}$ are found in only $0.8 \%$ of cases. Since the annual detection rate of TS1 pancreatic carcinoma or gastric cancer $(\leq 2 \mathrm{~cm})$ has remained at approximately $10 \%$ for the past 30 years, early detection and treatment have not been improved during the period [1]. Even when TS1 pancreatic carcinoma is detected and treated, $42 \%$ of patients are found in stage I with a 5 -year survival rate of less than $50 \%$ [2], which is regarded as an unsatisfactory result.

Diabetes, smokers, and chronic pancreatitis are known as high-risk group of pancreatic carcinoma [3] [4] [5] [6]. Periodic screening of patients with these risk factors is important for the early detection of pancreatic carcinoma. Tanaka et al. [7] reported that the dilatation of the main pancreatic duct by $2.5 \mathrm{~mm}$ or more detected by ultrasonography indicated high risk of pancreatic carcinoma. Clinicians should be aware of what modality to test for early detection of pancreatic carcinoma.

The aim of this study was to investigate methods for early detection of TS1 pancreatic carcinoma among patients undergoing surgery at our facility and to identify risk factors for recurrence after resection of the tumor.

\section{Materials and Methods}

\subsection{Patients}

Between April 2004 and March 2016, 444 patients with conventional pancreatic carcinoma underwent surgery at Tokai University Hospital and Tokai University Hachioji Hospital. Among these patients, the study targeted 65 patients (14.6\%) with a histopathological diagnosis of TS1 pancreatic carcinoma $(\leq 2 \mathrm{~cm})$. Special types of cancer, such as cancers derived from intraductal papillary mucinous neoplasms (IPMNs) or mucinous cystic neoplasms (MCNs), were excluded.

\subsection{Examination Items}

The study examined 65 cases of TS1 pancreatic carcinoma, recording data on the patient's background, reason for visiting the hospital, examination findings, histopathological findings, and treatment outcome. The UICC (Union for International Cancer Control), $7^{\text {th }}$ edition, was used to classify the level or stage of cancer.

\subsection{Statistical Analysis}

SPSS version 23 (IMB Japan, Tokyo, Japan) was used in all analyses. Survival rates were calculated by the Kaplan-Meier method. The chi-squared test was used for comparisons between groups. The Cox proportional hazards model was 
used for multivariate analysis. $P$ values $<0.05$ were considered to indicate a significant difference.

\section{Results}

\subsection{Patient Background}

The study included 65 patients, with a male-to-female ratio of 31:34, 52 to 86 years of age (average, 69 years) (Table 1). The site of the tumor was the pancreas head in 39 patients and the pancreatic body or tail in 26 patients. Pancreatoduodenectomy was performed in 38 patients, including portal vein resection in 8 patients. Duodenum-preserving pancreas head resection was performed in one patient because of a diagnosis of chronic pancreatitis. In 25 patients, the site of the tumor was the pancreatic tail, and distal pancreatectomy was performed in all of these patients (including 1 patient with portal vein resection).

\subsection{Reason for Hospital Visit}

On health examination, 26 patients (40.0\%) had symptoms and 39 patients (60.0\%) had no symptoms (Table 2). Sixteen of the 26 patients with symptoms had epigastric abdominal pain or dorsal pain. Six patients had jaundice, and two patients each had abdominal discomfort and weight loss. Abnormalities were found in 23 of the 39 patients with no symptoms at the time of health examination. Sixteen patients were found to have pancreatic carcinoma during follow-up for other disorders. The most frequent findings among the patients identified at the time of health examination were main pancreatic duct dilatation or tumor mass, which were detected in 13 patients by ultrasonography. Elevation of tumor marker (CA19-9) was found in three patients; elevation of hepatic and biliary enzymes, elevation of pancreatic enzymes, and impaired glucose tolerance were found in two patients each. Among the patients found to have pancreatic carcinoma during follow-up for other disorders, the most frequent disorders were diabetes in six patients and chronic pancreatitis in two patients. It was pointed out that five out of six patients for diabetic patients were exacerbation of diabetes.

\subsection{Examination Findings}

Although abnormal levels of CA19-9 were found in 33 patients (50.8\%), a high level of carcinoembryonic antigen (CEA) was found in only 16 patients $(24.6 \%)$ (Table 3). The rates of visualization of the tumor mass and of detection of main pancreatic duct dilatation by ultrasonography were $83.1 \%$ and $96.9 \%$, respectively. The rates of visualization of the tumor mass and of detection of main pancreatic duct dilatation by computed tomography (CT) were $66.2 \%$ and $95.4 \%$, respectively. The rate of visualization of the tumor mass by magnetic resonance imaging (MRI) was 56.9\%, and the rate of detection of main pancreatic duct dilatation by magnetic resonance cholangiopancreatography (MRCP) was 95.4\%. The rate of detection of main pancreatic duct dilatation by endoscopic 
Table 1. Patients background.

\begin{tabular}{cc}
\hline cases & 65 \\
sex (men/female) & $31 / 34$ \\
age (mean) & $52-86(69.0)$ \\
tumor-occupied site & 39 \\
head & 26 \\
body-tail & \\
operation & \\
pancreaticoduodenectomy & 38 (including 1 case for portal resection) \\
distal pancreatectomy & 25 (including 1 case for portal resection) \\
\hline
\end{tabular}

Table 2. Reasons for visit hospital.

\begin{tabular}{cccc}
\hline symptom & 26 cases $(40.0 \%)$ & no symptom & 39 cases $(60.0 \%)$ \\
\hline pain & 16 & health examination & 23 cases $(35.4 \%)$ \\
jaundice & 6 & ultrasonography & 13 \\
abdominal discomfort & 2 & elevation of tumor marker (CA19-9) & 3 \\
weight loss & 2 & elevation of hepatic and biliary enzyme & 2 \\
& & elevation of pancreatic enzyme & 2 \\
& impaired glucose tolerance & 2 \\
& follow-up for other disorders & 16 cases $(24.6 \%)$ \\
& diabetes & 6 \\
& chronic pancreatitis & 2 \\
& et al. & 8 \\
\hline
\end{tabular}

Table 3. Examination findings.

\begin{tabular}{ccc}
\hline modality & tumor mass & pancreatic duct dilatation \\
\hline US & $83.1 \%$ & $96.9 \%$ \\
CT & $66.2 \%$ & $95.4 \%$ \\
MRI & $56.9 \%$ & $95.4 \%$ \\
ERCP & - & $95.7 \%$ \\
EUS & $92.9 \%$ & $100 \%$ \\
\hline
\end{tabular}

retrograde cholangiopancreatography (ERCP) was $95.7 \%$. The rate of detection of class IV or $\mathrm{V}$ tumors by brush cytology was $45.2 \%$. The rates of visualization of the tumor mass and of detection of main pancreatic duct dilatation by endoscopic ultrasound (EUS) were $92.9 \%$ and $100 \%$, respectively.

\subsection{Histopathological Findings}

Tumor size ranged from 1 to $20 \mathrm{~mm}$ (average, $16.3 \mathrm{~mm}$ ), including seven tumors $\leq 10 \mathrm{~mm}$ (Table 4). There were $20 \mathrm{~T} 1$ tumors and $45 \mathrm{~T} 3$ tumors. Seventeen patients $(26.2 \%)$ were positive for lymph node metastasis: stage $1 \mathrm{~A}, 17$ patients 
Table 4. Histopathological findings.

\begin{tabular}{|c|c|c|}
\hline size & \multicolumn{2}{|c|}{$1-20$ mm (mean 16.3) } \\
\hline \multirow[t]{5}{*}{$\mathrm{T}$} & Tis & 0 \\
\hline & $\mathrm{T} 1$ & $20(30.8 \%)$ \\
\hline & $\mathrm{T} 2$ & 0 \\
\hline & $\mathrm{T} 3$ & $45(69.2 \%)$ \\
\hline & $\mathrm{T} 4$ & 0 \\
\hline \multirow[t]{2}{*}{$\mathrm{N}$} & No & $48(73.8 \%)$ \\
\hline & N1 & $17(26.2 \%)$ \\
\hline \multirow[t]{7}{*}{ Stage } & Stage 0 & 0 \\
\hline & Stage IA & $17(26.2 \%)$ \\
\hline & Stage IB & 0 \\
\hline & Stage IIA & $31(47.7 \%)$ \\
\hline & Stage IIB & $17(26.2 \%)$ \\
\hline & Stage III & 0 \\
\hline & Stage IV & 0 \\
\hline
\end{tabular}

(26.2\%); stage $2 \mathrm{~A}, 31$ patients (47.7\%); stage $2 \mathrm{~B}, 17$ patients $(26.2 \%)$.

\subsection{Treatment Outcomes}

The median survival time for the 65 patients with TS1 pancreatic carcinoma was 29.7 months; the 1-, 2-, and 5-year survival rates were $89.2 \%, 61.5 \%$, and $23.1 \%$, respectively. Twenty-eight patients (43.1\%) had a recurrence of cancer; the median period from operation to recurrence was 345 days (range, 75 to 1794 days). The site of recurrence was $24.6 \%$ local, $9.2 \%$ hepatic, $4.6 \%$ pulmonary, and $3.1 \%$ peritoneal. We divided the patients into a recurrence group and a nonrecurrence group and compared their histopathological characteristics (Table 5). On univariate analysis, the recurrence group had significantly higher rates of retroperitoneal tissue invasion, pancreatic neural invasion, venous invasion, lymphatic invasion, and lymph node metastasis. There were no significant differences between the groups in histological grade or residual tumor. In multivariate analysis, pancreatic neural invasion was an independent risk factor for recurrence (odds ratio, 6.333; 95\% CI, $1.834-21.872 ; p=0.004$ ).

\section{Discussion}

Although pancreatic carcinoma generally has a poor prognosis, the 5-year survival rate is $80.4 \%$ for cases in which the tumor had a diameter $\leq 10 \mathrm{~mm}$ at diagnosis [1]. Thus, early detection is important to improve the prognosis of pancreatic carcinoma [1]. However, most pancreatic carcinomas are detected as progressive cancers, even with current advanced diagnostic imaging systems. The purpose of this study was to present a clinically valuable method of verifying 
Table 5. Histopathological factors.

\begin{tabular}{cccccc}
\hline & \multicolumn{3}{c}{ univariate analysis } & \multicolumn{2}{c}{ multivariate analysis } \\
\hline & recurrence group & non-recurrence group & $p$ Value & RR $(95 \% \mathrm{CI})$ & $p$ Value \\
\hline $\mathrm{CH}+$ & 28 & 37 & & & \\
$\mathrm{DU}+$ & 1 & 5 & 0.304 & & \\
$\mathrm{~S}+$ & 8 & 1 & 0.68 & & \\
$\mathrm{RP}+$ & 19 & 6 & 0.185 & \\
$\mathrm{PV}+$ & 7 & 16 & 0.042 & & \\
$\mathrm{~A}+$ & 0 & 4 & 0.12 & & \\
$\mathrm{PL}+$ & 3 & 1 & 0.569 & & \\
$\mathrm{~N}+$ & 11 & 2 & 0.368 & & \\
$\mathrm{ne}$ & 24 & 6 & 0.035 & & \\
ly & 22 & 18 & 0.002 & $6.333(1.834-21.872)$ & \\
$\mathrm{v}$ & 22 & 18 & 0.013 & & \\
\hline
\end{tabular}

$\mathrm{CH}$; Bile duct invasion, DU; Duodenal invasion, S; Serosal side of the anterior pancreatic tissue invasion, RP; Retropancreatic tissue invasion, PV; Portal venous system invasion, A; Arterial system invasion, PL; Extrapancreatic nerve plexus invasion, $\mathrm{N}$; lymph node metastasis, ne; neural invasion, ly; lymphatic invasion, v; venous invasion.

early detection of TS1 pancreatic carcinoma by clarifying patient characteristics and their reasons for visiting the hospital.

It is difficult to determine whether a person develops pancreatic carcinoma due to diabetes or vice versa. However, in clinical practice we often find pancreatic carcinoma in cases of worsening diabetes. A meta-analysis of 88 papers found that the risk of pancreatic carcinoma was 1.97 times greater in diabetic patients than in nondiabetic patients. Therefore, a person with onset of diabetes within 1 year would have a higher risk of pancreatic carcinoma (6.69 times) than a 10 year-diabetic patient (1.36 times) [3]. In our study, 8 of 39 patients with no symptoms $(20.5 \%)$ had diabetes or impaired glucose tolerance. In addition to diabetes, smoking and obesity are risk factors for pancreatic carcinoma [4]. A relation between chronic pancreatitis and pancreatic carcinoma has also been pointed out for years, and the prevalence rate can be extremely high (6.9 to 16.5 times) [5] [6]. We report only two cases of chronic pancreatitis in this study, but chronic pancreatitis can be a high risk factor for onset of pancreatic carcinoma. These findings suggest that persons with diabetes or chronic pancreatitis, which are risk factors for pancreatic carcinoma, should undergo periodic screening for early detection of pancreatic carcinoma.

Ultrasonography is a useful screening method with low cost and low invasion. The rate of visualization of the tumor mass with abdominal ultrasonography for TS1 pancreatic carcinoma was $83.1 \%$, but the rate of detection of main pancreatic duct dilatation was extremely high at $96.9 \%$. Tanaka et al. [7] reported that dilatation of the main pancreatic duct by $2.5 \mathrm{~mm}$ or more detected by ultraso- 
nography indicated a high risk of pancreatic carcinoma. For other modalities, the rate of visualization of the tumor mass with CT and MRI was approximately $60 \%$, but it was over $90 \%$ for dilatation of the main pancreatic duct. It was also reported that indirect findings were recognized, such as dilatation of the main pancreatic duct found in $88 \%$ of cases of pancreatic carcinoma ( $\leq 20 \mathrm{~mm}$ ) [8]. $\mathrm{CT}$ and MRI may be useful for detailed examination, but not for screening because of their costs and the risk of radiation exposure. Since main pancreatic duct dilatation can be found accidentally during follow-up for other disorders, it is crucial to provide an appropriate detailed examination to avoid overlooking the disorder.

Pancreatic enzymes, such as traditional tumor markers (e.g., CA19-9, CEA), amylase, elastase, and DUPAN-2, are of limited usefulness for early diagnosis of pancreatic carcinoma. CA19-9 can be helpful for distinguishing good from bad tumors, determining resectability, predicting prognosis, and monitoring therapeutic effect, but it is less useful for early diagnosis [9] [10]. The positive rates of CEA and CA19-9 in TS1 pancreatic carcinoma are only $24.6 \%$ and $50.8 \%$, respectively. Recent studies reported that mutation of microRNAs could be useful to detect pancreatic carcinoma [11] [12], and clinical applications of this method are expected in the future.

EUS may be the most effective method for detailed examination of a patient who has shown an abnormal result in the screening process. The rates of visualization of the tumor mass and of detection of main pancreatic duct dilatation with EUS were $92.9 \%$ and $100 \%$, respectively. Maguchi et al. [13] reported that the tumor detection rate of TS1 pancreatic carcinoma $(\leq 2 \mathrm{~mm})$ was $100 \%$. Diagnosis by EUS-guided fine needle aspiration (EUS-FNA) at the time of tumor detection had a sensitivity of $85 \%$ to $94 \%$, a specificity of $100 \%$, and an accuracy of $95 \%$ [14] [15]. However, we should keep in mind that the accuracy would be lower for tumors $\leq 1 \mathrm{~mm}$ [16]. ERCP detected almost all cases of dilatation of the main pancreatic duct. However, the sensitivity and specificity of this method were not high, and there were frequent false-positive cases [17]. The positive rate of abrasive cytology was $45.2 \%$ in our study, and when considering complications such as pancreatitis in ERCP, the necessity for execution of ERCP in TS1 pancreatic carcinoma would be lower.

The reason for the extremely poor prognosis of pancreatic carcinoma is the high recurrence rate after curative resection [18]. Sperti et al. [19] reported a 2 -year recurrence rate of $95 \%$, with rates of $71.8 \%$ for local recurrence and $61.5 \%$ for hepatic recurrence. Other studies have reported rates of local and hepatic recurrence of $87 \%$ to $92 \%$ and $66 \%$ to $92 \%$, respectively [20] [21] [22]. We found a recurrence rate of TS1 pancreatic carcinoma of $43.1 \%$, which was lower than the overall rate for pancreatic carcinoma. The rates of local and hepatic recurrence were $24.6 \%$ and $9.2 \%$, respectively. Pancreatic neural invasion was an independent histopathological risk factor for recurrence (odds ratio, 6.333; 95\% CI, 1.834 - 21.872; $p=0.004)$. Reported risk factors for recurrence of pancreatic carcinoma include stage of progression, tumor size, tissue type, and residual tu- 
mor [23] [24] [25]. However, we have found no published reports on risk factors for recurrence of small pancreatic carcinomas. The results of this study demonstrate the importance of local control by surgery as well as control of local recurrence by adjunctive therapy in cases of TSI pancreatic carcinoma.

\section{Conclusion}

To screen for TS1 pancreatic carcinoma, the study first examined the main pancreatic duct dilatation by ultrasonography and then conducted a detailed examination using endoscopic ultrasound. Patients with pancreatic neural invasion require careful attention for local recurrence.

\section{Acknowledgements}

The authors would like to thank Enago for the English language review.

\section{Conflict of Interest Statement}

Hideki Izumi and other co-authors have no conflict of interest.

\section{References}

[1] Egawa, S., Toma, H., Ohigashi, H., Okusaka, T., Nakao, A., Hatori, T., et al. (2012) Japan Pancreatic Cancer Registry; 30th Year Anniversary: Japan Pancreas Society. Pancreas, 41, 985-992. https://doi.org/10.1097/MPA.0b013e318258055c

[2] Tsunoda, T., Yamamoto, Y., Kimoto, M., Imai, H., Iwamoto, S., Kawasaki, S., et al. (1998) Staging and Treatment for Patients with Pancreatic Cancer. How Small Is an Early Pancreatic Cancer? Journal of Hepato-Biliary-Pancreatic Surgery, 5, 128-132. https://doi.org/10.1007/s005340050022

[3] Batabyal, P., Vander, Hoorn, S., Christophi, C. and Nikfarjam, M. (2014) Association of Diabetes Mellitus and Pancreatic Adenocarcinoma: A Meta-Analysis of 88 Studies. Annals of Surgical Oncology, 21, 2453-2462.

https://doi.org/10.1245/s10434-014-3625-6

[4] Larsson, S.C., Permert, J., Hakansson, N., Naslund, I., Bergkvist, L. and Wolk, A. (2005) Overall Obesity, Abdominal Adiposity, Diabetes and Cigarette Smoking in Relation to the Risk of Pancreatic Cancer in Two Swedish Population-Based Cohorts. British Journal of Cancer, 93, 1310-1315.

https://doi.org/10.1038/sj.bjc.6602868

[5] Bang, U.C., Benfield, T., Hyldstrup, L., Bendtsen, F. and Beck Jensen, J.E. (2014) Mortality, Cancer, and Comorbidities Associated with Chronic Pancreatitis: A Danish Nationwide Matched-Cohort Study. Gastroenterology, 146, 989-994. https://doi.org/10.1053/j.gastro.2013.12.033

[6] Lowenfels, A.B., Maisonneuve, P., Cavallini, G., Ammann, R.W., Lankisch, P.G., Andersen, J.R., et al. (1993) Pancreatitis and the Risk of Pancreatic Cancer. International Pancreatitis Study Group. The New England Journal of Medicine, 328, 1433-1437. https://doi.org/10.1056/NEJM199305203282001

[7] Tanaka, S., Nakao, M., Ioka, T., Takakura, R., Takano, Y., Tsukuma, H., et al. (2010) Slight Dilatation of the Main Pancreatic Duct and Presence of Pancreatic Cysts as Predictive Signs of Pancreatic Cancer: A Prospective Study. Radiology, 254, 965-972. https://doi.org/10.1148/radiol.09090992 
[8] Yoon, S.H., Lee, J.M., Cho, J.Y., Kyung, B.L., Ji, E.K., Seung, K.M., et al. (2011) Small ( $\leq 20 \mathrm{~mm}$ ) Pancreatic Adenocarcinomas: Analysis of Enhancement Patterns and Secondary Signs with Multiphasic Multidetector CT. Radiology, 259, 442-452. https://doi.org/10.1148/radiol.11101133

[9] Duffy, M.J., Sturgeon, C., Lamerz, R., Haglund, C., Holubec, V.L., Klapdor, R., et al. (2010) Tumor Markers in Pancreatic Cancer: A European Group on Tumor Markers (EGTM) Status Report. Annals of Oncology, 21, 441-447.

https://doi.org/10.1093/annonc/mdp332

[10] Goonetilleke, K.S. and Siriwardena, A.K. (2007) Systematic Review of Carbohydrate Antigen (CA 19-9) as a Biochemical Marker in the Diagnosis of Pancreatic Cancer. European Journal of Surgical Oncology, 33, 266-270. https://doi.org/10.1016/j.ejso.2006.10.004

[11] Yu, J., Li, A., Hong, S.M., Hruban, R.H. and Goggins, M. (2012) MicroRNA Alterations of Pancreatic Intraepithelial Neoplasias. Clinical Cancer Research, 18, 981-992. https://doi.org/10.1158/1078-0432.CCR-11-2347

[12] Kojima, M., Sudo, H., Kawauchi, J., Takizawa, S., Kondou, S., Nobumasa, H., et al. (2015) MicroRNA Markers for the Diagnosis of Pancreatic and Biliary-Tract Cancers. PloS ONE, 10, e0118220. https://doi.org/10.1371/journal.pone.0118220

[13] Maguchi, H. (2004) The Roles of Endoscopic Ultrasonography in the Diagnosis of Pancreatic Tumors. Journal of Hepato-Biliary-Pancreatic Surgery, 11, 1-3. https://doi.org/10.1007/s00534-002-0752-4

[14] Yamao, K., Sawaki, A., Mizuno, N., Shimizu, Y., Yatabe, Y. and Koshikawa, T. (2005) Endoscopic Ultrasound-Guided Fine-Needle Aspiration Biopsy (EUS-FNAB): Past, Present, and Future. Journal of Gastroenterology, 40, 1013-1023. https://doi.org/10.1007/s00535-005-1717-6

[15] Fisher, L., Segarajasingam, D.S., Stewart, C., Deboer, W.B. and Yusoff, I.F. (2009) Endoscopic Ultrasound Guided Fine Needle Aspiration of Solid Pancreatic Lesions: Performance and Outcomes. Journal of Gastroenterology and Hepatology, 24, 90-96. https://doi.org/10.1111/j.1440-1746.2008.05569.x

[16] Yamao, K., Ohashi, K., Mizutani, S., et al. (1998) Endoscopic Ultrasound-Guided Fine-Needle Aspiration (EUS-FNA) for the Diagnosis of Digestive Diseases. Endoscopy, 30, A176-A178. https://doi.org/10.1055/s-2007-1001513

[17] Wakatsuki, T., Irisawa, A., Bhutani, M.S., Hikichi, T., Shibukawa, G., Takagi, T., et al. (2005) Comparative Study of Diagnostic Value of Cytologic Sampling by Endoscopic Ultrasonography-Guided Fine-Needle Aspiration and That by Endoscopic Retrograde Pancreatography for the Management of Pancreatic Mass without Biliary Stricture. Journal of Gastroenterology and Hepatology, 20, 1707-1711. https://doi.org/10.1111/j.1440-1746.2005.03900.x

[18] Han, S.S., Jang, J.Y., Kim, S.W., Kim, W.H., Lee, K.U. and Park, Y.H. (2006) Analysis of Long-Term Survivors after Surgical Resection for Pancreatic Cancer. Pancreas, 32, 271-275. https://doi.org/10.1097/01.mpa.0000202953.87740.93

[19] Sperti, C., Pasquali, C., Piccoli, A. and Pedrazzoli, S. (1997) Recurrence after Resection for Ductal Adenocarcinoma of the Pancreas. World Journal of Surgery, 21, 195-200. https://doi.org/10.1007/s002689900215

[20] Griffin, J.F., Smalley, S.R., Jewell, W., Paradelo, J.C., Reymond, R.D., Hassanein, R.E., et al. (1990) Patterns of Failure after Curative Resection of Pancreatic Carcinoma. Cancer, 66, 56-61.

https://doi.org/10.1002/1097-0142(19900701)66:1<56::AID-CNCR2820660112>3.0. $\underline{\mathrm{CO} ; 2-6}$ 
[21] Kayahara, M., Nagakawa, T., Ueno, K., Ohta, T., Takeda, T. and Miyazaki, I. (1993) An Evaluation of Radical Resection for Pancreatic Cancer Based on the Mode of Recurrence as Determined by Autopsy and Diagnostic Imaging. Cancer, 72, 2118-2123.

https://doi.org/10.1002/1097-0142(19931001)72:7<2118::AID-CNCR2820720710>3. $0 . \mathrm{CO} ; 2-4$

[22] Westerdahl, J., Andren-Sandberg, A. and Ihse, I. (1993) Recurrence of Exocrine Pancreatic Cancer-Local or Hepatic? Hepatogastroenterology, 40, 384-387.

[23] Matsuno, S., Egawa, S., Fukuyama, S., Fuyuhiko, M., Makoto, S., Shuji, I., et al. (2004) Pancreatic Cancer Registry in Japan: 20 Years of Experience. Pancreas, 28, 219-230. https://doi.org/10.1097/00006676-200404000-00002

[24] Luttges, J., Schemm, S., Vogel, I., Hedderich, J., Kremer, B. and Kloppel, G. (2000) The Grade of Pancreatic Ductal Carcinoma Is an Independent Prognostic Factor and Is Superior to the Immunohistochemical Assessment of Proliferation. The Journal of Pathology, 191, 154-161.

https://doi.org/10.1002/(SICI)1096-9896(200006)191:2<154::AID-PATH603>3.0.C $\underline{\mathrm{O} ; 2-\mathrm{C}}$

[25] Neoptolemos, J.P., Stocken, D.D., Dunn, J.A., et al. (2001) Influence of Resection Margins on Survival for Patients with Pancreatic Cancer Treated by Adjuvant Chemoradiation and/or Chemotherapy in the ESPAC-1 Randomized Controlled Trial. Annals of Surgery, 234, 758-768.

https://doi.org/10.1097/00000658-200112000-00007 